\section{Géneros}

Multidisciplinary Journal of Gender: Studies

\section{Hipatia Press \\ www.hipatiapress.com}

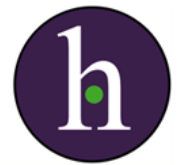

Instructions for authors, subscriptions and further details:

$\underline{\text { http://generos.hipatiapress.com }}$

\title{
Programas, Intervenciones y Prácticas Efectivas en Prevención de Drogodependencias con Mujeres Jóvenes
}

Rosario Pozo Gordaliza ${ }^{1}$

Carmen Orte ${ }^{1}$

Marga Vives ${ }^{1}$

1) Department of Education, Universitat de les Illes Balears

Date of publication: February $25^{\text {th }}, 2016$

Edition period: February - June 2016

To cite this article: Pozo, R.; Orte, C. \& Vives, M. (2016). Programas, Intervenciones y Prácticas Efectivas en Prevención de Drogodependencias con Mujeres Jóvenes. Multidisciplinary Journal of Gender Studies, 5(1), 859886. doi: 10.17583/generos.2016.1776

To link this article: http://dx.doi.org/10.17583/generos.2016.1776

\section{PLEASE SCROLL DOWN FOR ARTICLE}

The terms and conditions of use are related to the Open Journal System and

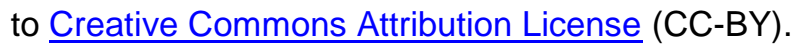




\section{Programas, Intervenciones y Prácticas Efectivas en Prevención de}

\section{Drogodependencias con Mujeres Jóvenes}

Rosario Pozo Gordaliza

Marga Vives

Carmen Orte

Universitat de les Illes Balears

\section{Resumen}

Recientemente a nivel internacional se ha incrementado el interés en la identificación de prácticas efectivas que contemplen las necesidades particulares de las niñas o jóvenes, precisamente motivado por el abuso de sustancias tóxicas y sus "altos" contactos con Justicia juvenil y de adultos (Rosenbaum, 2002 entre otros). La literatura revisada revela que en general son muy escasos los programas específicos para chicas en materia de prevención de drogodependencias. Además son también pocos los que han publicado sus resultados desagregados por sexo. Por lo que la necesidad de estudio y de evaluación son tremendamente necesarios para poder dar una respuesta adecuada a sus necesidades. Por todo ello, este artículo revisa y analiza los distintos discursos entorno a los factores de éxito y riesgo al igual que los programas específicos en prevención de drogodependencias (y residenciales) con chicas o mujeres jóvenes.

Palabras clave: jóvenes infractoras, programas basados en evidencia, residenciales, drogodependencias. 


\section{Programs, Interventions and Effective Practices in Drug Prevention with Young Women and Girls}

Rosario Pozo Gordaliza

Marga Vives

Carmen Orte

Universitat de les Illes Balears

\section{Abstract}

Recently at the international level has been increased the interest in identifying effective practices that address the particular needs of young girls. This was motivated precisely by the high contact of girls who consume drugs and have contact with the juvenile Justice System. The literature review reveals that in general there are very few specific programs who address girls needs in the prevention of drug addiction. In addition are also very few who have published their results disaggregated by sex. So the need for study and evaluation are extremely necessary to provide an adequate response to their needs. Therefore, this paper reviews and analyzes the different discourses around the success and risk factors as well as the specific programs in drug prevention with girls and young women.

Keywords: young offenders, evidence based programs, drug addiction. 


\section{Pozo, Vives \& Orte - Prevención de Drogodependencias}

$\mathrm{E}$

xiste numeroso material documental que habla sobre chicos jóvenes, delincuencia y drogodependencias, pero casi nunca, o en contadas ocasiones mencionan a las chicas. Según apuntaba Worrall (2001), este es un grupo que hasta ahora ha sido valorado como demasiado pequeño y con "bajo riesgo" como para prestarle atención. Los primeros estudios acerca de la delincuencia juvenil femenina estuvieron (re) cargados de androcentrismo y negligencia. La mayor parte de las teorías estaban desarrolladas para comprender la etiología de la delincuencia masculina ${ }^{1}$, por lo que las líneas de intervención al respecto estaban pensadas desde y para la óptica masculina pero no necesariamente la femenina. Según apuntaba acertadamente Worrall (2001), las jóvenes que delinquían (y consumían tóxicos) han sido definidas históricamente de distinta forma que los chicos $^{2}$. En general, mientras las chicas han sido consideradas penalmente menos inclinadas al delito que sus iguales varones, el comportamiento de aquellas que violan los estereotipos de género (como consumir o delinquir) de conformidad y docilidad han dado lugar, como consecuencia, a la creencia de que éstas tienen un mayor potencial patológico (Gelsthorpe, 1989). Así mismo, Gaarder, Rodríguez y Zatz (2004) encontraron en sus investigaciones una falta de información y formación de las personas que trabajaban con las jóvenes sobre todo en el sistema de justicia juvenil en términos de estereotipos raciales y de género, lo que limitaba a que las jóvenes pudieran acceder adecuadamente a los servicios necesarios. Una pregunta ineludible que debemos hacernos es ¿qué relación tienen las drogas en el estudio de la delincuencia juvenil femenina?. En primer lugar, hay que señalar que los trabajos precedentes sobre drogas y mujeres los encontramos en el año 1979, cuando el Instituto Nacional sobre Abuso de Drogas o NIDA del Gobierno norteamericano señaló que: los estudios han demostrado que las mujeres difieren de los hombres en sus tasas y patrones de abuso de drogas, y llamó la atención el estigma particular que ocasionaba el abuso de drogas femenino (Burt, Glynn \& Sowder, 1979). El mismo estudio apuntaba, igualmente, la necesidad de hacer investigaciones específicas en este sentido. Asimismo, Glynn, Leukefeld, y Ludford (1983) señalaban que a medida que la atención sobre el campo del abuso de drogas comenzó a expandirse más allá del consumo de opiáceos, los/as investigadores/as comenzaron a reportar interesantes descubrimientos que sugirieron cómo los patrones de consumo de drogas de las mujeres no 
pueden establecerse simplemente generalizando de los estudios sobre los de los hombres. En su lugar, comenzaron a surgir trabajos que reconocían como muchos de los problemas de las mujeres consumidoras no eran menores, iguales o superiores a los de los hombres consumidores, sino que eran propios de las consumidoras, y era necesario explicarlo desde su propio marco de referencia Glynn, Wallenstein Pearsons y Sayers (1983). Por lo que considerar el género como algo neutral o ser ciegos ante él ha provocado que esto tenga un impacto negativo en las chicas y las mujeres. A las jóvenes se las ha ignorado y mal investigado. Han sido definidas y estudiadas por su sexo, no por su género. Esto ha provocado una distorsión en la fotografía. Además, la historia reciente demuestra que ha habido una clara desatención en el uso y abuso de sustancias tóxicas, en la delincuencia y los problemas de salud mental de las niñas y mujeres jóvenes (NATIONAL INSTITUTE ON DRUG ABUSE, 1979 y 2002). Entender el contexto de las niñas y su delincuencia/consumo requiere no sólo un conocimiento de su historia, también una revisión de su desarrollo evolutivo y adolescente. Esto es necesario tenerlo en cuenta puesto que sin este análisis difícilmente comprenderemos la situación actual de negligencia en cuanto a investigaciones y programas específicos de intervención de prevención y tratamiento y la invisibilización de las jóvenes dentro y fuera del sistema de Justicia juvenil y los programas de drogodependencias (motivado en cierta manera por su escasa o reducida presencia) ha sido una constante, y aún sigue siendo una asignatura pendiente (Sharpe \& Gelsthorpe, 2009). Como bien explicaba Chesney-Lind en muchas de sus obras, una teoría de las jóvenes tiene que dar información sobre sus vidas porque esencialmente poco se sabe sobre las chicas. Este reducido número de mujeres jóvenes consumidoras y en contacto con el sistema de justicia juvenil no indica precisamente pequeños problemas, sino que, en ocasiones, se justifica o se deniega su acceso a que sea investigado, a programas o servicios específicos. Al igual que les ocurrió a las mujeres adultas en otras épocas, su número reducido ha invitado a nuevas negligencias y nuevas formas de abuso. En la actualidad, las jóvenes están en el punto de mira porque ha habido un incremento significativo en su inicio y abuso de sustancias tóxicas desde el año 1992, que coincide con el aumento de su detención y encarcelamiento Kumpfer, Smith y Summerhays (2008). Como resultado, muchos/as investigadores/as y profesionales han comenzado a prestar una especial 


\section{Pozo, Vives \& Orte - Prevención de Drogodependencias}

atención a esta cuestión. Ha costado reconocer, y aún cuesta, que muchos de los múltiples problemas que presentan las jóvenes (ver, por ejemplo, Rumgay, 1998; Chesney-Lind, 1997; Cameron \& Telfer, 2004). son en parte compartidos, pero no iguales y, asimismo, diferenciales por motivos de edad, género, etnicidad y clase socioeconómica/cultural. El género es relevante a la hora de explicar cómo las jóvenes se implican en el consumo y continúan inmersas en procesos delincuenciales. La información de la que hasta la fecha se dispone permite observar que las diferencias de género en la socialización y el desarrollo, en el inicio y continuidad en el consumo realmente existen y que estas diferencias tienen un efecto en los patrones de la delincuencia. A pesar de que chicos y chicas comparten algunas de las mismas presiones y situaciones de vulnerabilidad y de desventaja social en sus vidas y a veces el mismo acceso al aprendizaje del comportamiento delincuente o de consumo ${ }^{3}$. Las razones por las cuales chicos y chicas se involucran en actos delincuenciales o de consumo — además de las formas de llegada o entrada a las instituciones de protección y de reforma entre otros - son diferentes (véase anexo 1). Chicos y chicas no tienen las mismas oportunidades "reales", ya que existe en la estructura social una doble moral acerca de cómo deben comportarse hombres y mujeres, niños y niñas, los/as jóvenes, por lo que es fácil pensar que también, al igual que en sus vidas, las chicas tengan maneras diferentes de consumir y de delinquir. Los datos de los distintos estudios apuntan a una fuerte relación entre la victimización, delincuencia y traumas en las jóvenes (Belknap \& Holsinger, 1998; Chesney-Lind \& Shelden, 1992, 1997, 2004). La investigación sobre historias de abuso sexual infantil de los delincuentes juveniles y adultos sugiere que las tasas de abuso sexual son mayores para las niñas que para los niños (Dembo et al, 1993). En un estudio con niñas y mujeres jóvenes encarceladas en el California Youth Authority, Bloom, Owen, Piper Deschenes y Rosenbaum (2002) encontraron que tres cuartas partes de las mujeres jóvenes reportaron abuso físico continuado, y casi la mitad (46\%) sufrieron abuso sexual. A menudo, el abuso en el hogar provoca fugas continuadas o intermitentes y las estrategias de las jóvenes, en la calle, no están exentas de una cultura patriarcal que las relega a una situación de inferioridad, de modo que muchos de los comportamientos de las jóvenes son respuestas a la victimización en situaciones de exclusión social. Esta situación se agudiza entre las clases socioeconómicas culturales más 
empobrecidas, en las que existe una clara diferenciación de roles. La huida del hogar es uno de los factores de riesgo más frecuentes para las niñas de involucrarse en un consumo o con el sistema de justicia de menores y en última instancia puede conducir a su encarcelamiento. Los datos de arrestos indican que las niñas y los niños que han protagonizado fugas de sus casas es aproximadamente el mismo número pero que las niñas son detenidas con más frecuencia que los varones (Chesney-Lind \& Shelden, 1997). Otra idea esencial para comprender este fenómeno es que los aumentos en detenciones por "delitos violentos" en jóvenes no son necesariamente debido a un aumento significativo en su comportamiento violento, pero si es una manera de redefinir su comportamiento como conflictivo o de delito violento. Un ejemplo de ello es que los estudios que usan auto-informes delincuenciales difieren significativamente de los registros oficiales (Huizinga, 1997). Habitualmente las razones por las cuales chicas chicos agreden son diferentes. Hay que recordar que ellas conviven con violencia en todos sus espacios de referencia colegios, familia, barrio, grupo de iguales etc. y suelen pelearse de forma puntual con una persona cercana dentro de las relaciones interpersonales (fundamentalmente en espacios de lo privado). Sin duda el consumo está relacionado con esas agresiones. Aunque es preciso destacar que el consumo "problemático" es un problema más que se añade a su biografía. No es necesariamente el motivo principal por el cual se encuentran en contacto con Justicia juvenil. En términos generales suelen participar menos en delitos faltas o infracciones graves o violentas y tienen más probabilidades de estar involucradas en delitos, infracciones o faltas contra la propiedad o tráfico de drogas. Además, en numerosas ocasiones los contactos con el sistema de justicia juvenil son debido a que los controles sociales informales mencionados anteriormente acuden a estas instancias informando de la situación. El aumento de arrestos se traduce sin duda a un mayor impacto en su institucionalización en el sistema de justicia de menores.

\section{Programas de intervención con niñas y jóvenes ¿aproximación sensible o específica de género?}

La literatura sobre el fenómeno nos habla de dos tipologías o corrientes diferenciales para abordar el fenómeno. En primer lugar, existe la 
denominada específica de género con una perspectiva crítica feminista entorno al machismo y al paternalismo del sistema de justicia juvenil y dirige su atención a cómo mejorar la prevención de la delincuencia femenina y su compleja diversidad ${ }^{4}$ (Brown, 2003; Belknap, 2001; Howell, 2003), o "sensibles de género" basadas en revisiones cuantitativas de los estudios sobre programas específicos en términos de reincidencia (Cullen \& Gendreau, 2000; Gendreau, 1996 y otros $)^{5}$. Además es necesario referenciar que la prevención universal hace referencia a las estrategias dirigidas a toda la población, escolar o de cualquier otro grupo, con objeto de prevenir o retrasar el consumo de drogas. En cambio la prevención selectiva hace referencia a las estrategias dirigidas a grupos específicos que tienen un riesgo mayor que otros de tener problemas relacionados con las drogas. La mayor parte de los programas que recogemos se basan en este modelo tratando de prevenir el consumo de drogas mediante el fortalecimiento de los factores protectores, como, por ejemplo, la autoestima y desarrollo de habilidades sociales o resolución de conflictos entre otros. Las autoras Belknap, (2001) y Chesney-Lind, (2000) señalan que los programas no pueden ni deben centrarse únicamente en los varones ni pueden demostrar su utilidad en términos de programación para las niñas a partir de modelos basados empíricamente en varones. Tanto las niñas que ingresan en el sistema de justicia de menores como aquellas que reciben programas de prevención en drogodependencias, a menudo se encuentran con que los programas en los cuales participan se han creado y son para o mantienen una racionalidad masculina destinado principalmente a los niños/as delincuentes, potenciales o consumidores de tóxicos. Si hacemos un esfuerzo de recuerdo en un pasado reciente, tanto el mundo de la drogodependencia como el delincuencial son espacios tradicionalmente vinculados al espacio de lo público y por lo tanto masculinizado. Existen numerosos programas preventivos en drogodependencias y delincuenciales con resultados positivos para los chicos sobre todo los basados en la prevención en la comunidad. Aunque escasos, algunos estudios como por ejemplo CSAP Community Partnership Cross-Site Study The 48-Community Partnerships Cross-Site Study (Yin \& Ware, 2000), señalan que encontraron efectos positivos en varones pero no necesariamente en chicas y en algunos casos incluso resultados negativos para ellas. Una serie de programas han demostrado éxito en la reducción y la iniciación del consumo del tabaquismo de las 
niñas $^{6}$ (Blake et al, 2001) (y además son los que concentran sus esfuerzos en aspectos como las relaciones familiares, la supervisión parental, la comunicación y tienen en cuenta el estrés, la depresión, la seguridad, y la imagen del cuerpo entre otros), sin embargo, sólo un par de programas han demostrado el éxito en sustancias como el alcohol y la marihuana. En los factores de protección para las niñas y jóvenes (ver anexo1), cuando la supervisión de los padres y madres es alta, las adolescentes tenían menos probabilidades para desarrollar un consumo de sustancias que los chicos. Además, las actitudes de los padres y madres hacia el consumo y la vinculación a la escuela tuvo un impacto mayor en las niñas. Por lo que los programas de prevención basados en competencias familiares de cuidado y de supervisión parecen, a priori, tener una influencia ligeramente mayor en las niñas que en los varones ${ }^{7}$, mientras que los programas de tipo comunitario o de auto-control tenían una mayor influencia en los varones (Kumpfer \& Alvarado, 2003). A lo largo de estos años se han desarrollado un buen número de programas preventivos considerados como efectivos en distintos sitios web y agencias estatales de prevención. Sin embargo muchos de ellos aun siendo replicados escasean estudios rigurosos publicados sobre sus efectos en términos de validez interna o externa (Kumpfer, Smith \& Summerhays, 2008 entre otros). A continuación presentamos una tabla con los programas adaptados o específicos para chicas: 
Tabla 1

Programas efectivos en prevención de drogodependencias y otras conductas problemáticas en niñas jóvenes.

\section{PREVENCIÓN GENERAL}

\begin{tabular}{|c|c|c|}
\hline Programas & Descripción & Observaciones \\
\hline $\begin{array}{l}\text { CAMH } \\
\begin{array}{l}\text { (www.camh.net/Publicat } \\
\text { Resources_for_Professic } \\
\text { Validity/validity } \\
\text { reframing.html) }\end{array}\end{array}$ & $\begin{array}{l}\text { Novedoso "para y por la juventud" que considera las voces de } \\
\text { niñas y las mujeres jóvenes. El proyecto va más allá del discurso } \\
\text { sanitario y trata de generar pautas comprensivas de qué es la } \\
\text { depresión }{ }^{8} \text { y anima a compartir conocimientos y percepciones } \\
\text { sobre los factores de riesgo y de protección. El objetivo es } \\
\text { prevenir la depresión incidiendo en las actividades físicas e } \\
\text { intelectuales. Interrelaciona la depresión, autoestima, imagen } \\
\text { corporal positiva, reducción de stress e incidiendo en los medios } \\
\text { de comunicación para el empoderamiento de las mismas. }\end{array}$ & $\begin{array}{l}\text { No aportan datos precisos sobre la } \\
\text { evaluación. } \\
\text { Uno de los puntos fuertes que } \\
\text { desarrolla es el apoyo y la escucha } \\
\text { entre el grupo de iguales. }\end{array}$ \\
\hline $\begin{array}{l}\text { Girls Inc (1992) } \\
\text { www.girlsinc-online.org }\end{array}$ & $\begin{array}{l}\text { Tiene un fuerte componente virtual y desarrolla ejercicios } \\
\text { mentales de concentración, conocer su propio cuerpo y la } \\
\text { planificación para el futuro entre otros. Desarrolla habilidades } \\
\text { sociales mediante recursos educativos y de auto-defensa. Para } \\
\text { que las chicas asuman riesgos, refuerzo escolar, prevención de } \\
\text { embarazos, violencia y abuso de drogas mediante el desarrollo } \\
\text { mental y físico. }\end{array}$ & $\begin{array}{l}\text { No aportan datos precisos sobre la } \\
\text { evaluación. } \\
\text { Punto fuerte, alude a que esta } \\
\text { iniciativa está basada en una } \\
\text { investigación }\end{array}$ \\
\hline
\end{tabular}




\begin{tabular}{|c|c|c|}
\hline Programas & Descripción & Observaciones \\
\hline $\begin{array}{l}\text { Family connection } \\
\text { Computer-delivered } \\
\text { substance use preventior } \\
\text { program for adolescen1 } \\
\text { girls and their mothers. } \\
\text { Schinke et al (2010) }\end{array}$ & $\begin{array}{l}\text { Programa de prevención del consumo de sustancias dirigido a } \\
\text { mujeres adolescentes y a sus madres que busca identificar } \\
\text { aquellos factores de riesgo y de protección que contribuyan al } \\
\text { inicio y abuso de sustancias tóxicas mediante el fortalecimiento } \\
\text { de las interacciones madre-hija. En una semana madres e hijas } \\
\text { trabajan juntas para completar una sesión de } 45 \text { minutos en el } \\
\text { ordenador consistentes en ejercicios interactivos para centrarse } \\
\text { en temas como el desarrollo de habilidades de comunicación, } \\
\text { imagen personal, y el uso de sustancias tóxicas. }\end{array}$ & $\begin{array}{l}\text { Los beneficios se observaron en } \\
\text { el segundo año de seguimiento, } \\
\text { tanto para las hijas como para } \\
\text { sus madres. La intervención } \\
\text { reportó menores factores de } \\
\text { riesgo y más altos factores de } \\
\text { protección, y sus madres } \\
\text { reportaron menor consumo de } \\
\text { alcohol. }\end{array}$ \\
\hline
\end{tabular}

\section{PREVENCIÓN SELECTIVA}

\begin{tabular}{lll}
\hline Programas & Descripción & Observaciones \\
\hline & Programa de prevención basado en la evidencia científica que \\
Community connection & involucra a todos los sectores de la comunidad en la promoción Las jóvenes desarrollan un papel \\
Communities That Cart del desarrollo positivo de la juventud. El objetivo es reducir activo en la comunidad (de & (ww.ctcsquamish.com) & $\begin{array}{l}\text { riesgos y aumentar los factores de protección para los niños y los liderazgo entre las mujeres } \\
\text { jóvenes, y fortalecer vínculos en el hogar, la escuela, entre el jóvenes). } \\
\text { grupo de iguales y la comunidad. }\end{array}$ \\
\hline
\end{tabular}


Gender-specific treatmer

Youth Solvent Addictior Identifica cinco áreas de prevención: el abuso de sustancias, Committee

(www.ccsa.ca/eng/part nerships/ysac/Pages/de fault.aspx)

\section{GRUPOS CONTROL}

\section{Programas}

Lei-'Ilima-Health

(Kameoke, 1996).

Conexiones para

mujeres jóvenes adolesc r educación preventiva. delincuencia, embarazos no deseados de adolescentes, dificultades académicas, la violencia y la depresión. El objetivo es el fortalecimiento de familias (padres y sus hijos).

\section{Descripción}

Resultados positivos en alcanzar los objetivos que se propone. Punto fuerte; desarrolla un enfoque de género en todos los niveles de tratamiento de la juventud de origen aborigen.
El Strengthening Hawaiian Families Program fue implementado descenso de los ratios de depresion, en los colegios y evaluado por investigadores de la Universidad de incremento de satisfacción en la Hawaii. La intervención se basó en: -Escuela primaria -Clases de apariencia física, incremento de habilidades de comunicación en el uso de tabaco y de drogas.

Incremento de la autoestima, de las estrategias para la toma de decisiones, habilidades sociales, relaciones de confianza positiva entre madre e hija. También aportó un descenso de consumo de sustancias en 30 días y en los ratios de inicio. 


\begin{tabular}{|c|c|c|}
\hline Programas & Descripción & Observaciones \\
\hline PEERsuasion & $\begin{array}{l}\text { Desarrollo del liderazgo y empoderamiento de las niñas y chicas } \\
\text { pertenecientes a minorías étnicas. }\end{array}$ & $\begin{array}{l}\text { En comparación con el grupo de } \\
\text { control, las chicas que } \\
\text { participaban en el programa } \\
\text { específico de género reportaban } \\
\text { índices más bajos de inicio y } \\
\text { abuso en el consumo de alcohol. }\end{array}$ \\
\hline $\begin{array}{l}\text { ATHENA } \\
\text { Elliot et al. (2002) }\end{array}$ & $\begin{array}{l}\text { Desarrollaron un programa específico de género de la versić } \\
\text { ATLAS. Esta versión adaptada se centraba en mejorar la nutrición } \\
\text { el ejercicio físico para prevenir el consumo y los trastornc } \\
\text { alimenticios. }\end{array}$ & $\begin{array}{l}\text { Los hallazgos fueron que } \\
\text { comparado con un grupo que no ha } \\
\text { tenido un tratamiento las chicas } \\
\text { aumentaron sus conocimientos } \\
\text { sobre las drogas y reducción de su } \\
\text { consumo. }\end{array}$ \\
\hline RYSE & $\begin{array}{l}\text { Programa específico de género sensible culturalmente con el } \\
\text { colectivo afroamericano. El objetivo general es no reincidir y } \\
\text { volver a los centros de internamiento del sistema de Justicia } \\
\text { Juvenil. Para ello tiene en cuenta múltiples factores (familia, } \\
\text { colegio, grupo de iguales) y ambos incorporan planes de } \\
\text { tratamiento individualizados. }\end{array}$ & $\begin{array}{l}\text { Parece que funciona mejor para las } \\
\text { afroamericanas y las hispanas. } \\
\text { Los resultados muestran menos } \\
\text { arrestos y cuando estos se } \\
\text { producían eran menos graves. } \\
\text { Además de mejoras educativas } \\
\text { (Atención). }\end{array}$ \\
\hline
\end{tabular}




\begin{tabular}{|c|c|c|}
\hline Programas & Descripción & Observaciones \\
\hline The WINGS & $\begin{array}{l}\text { Para evaluar su efectividad se hizo una investigación con grupos } \\
\text { control y se midió la reincidencia y el producto educativo (éxito } \\
\text { escolar, fracasos y expulsiones. Autocontrol, y actitudes } \\
\text { prosociales), relaciones con adultos, manejo de la presión social } \\
\text { entre el grupo de iguales, entre otros. }\end{array}$ & $\begin{array}{l}\text { Aunque a priori se detectaron } \\
\text { mejoras en reducción de } \\
\text { amistades delincuentes, hábitos de } \\
\text { stress, presión entre grupo de } \\
\text { iguales, soledad social, consumo } \\
\text { de drogas etc. A largo plazo se } \\
\text { detectó que no reducían los ratios } \\
\text { de actividades delincuentes. }\end{array}$ \\
\hline \multicolumn{3}{|c|}{ DISEÑOS (SIN GRUPO DE CONTROL (PRE TEST Y POST TEST) } \\
\hline Programas & Descripción & Observaciones \\
\hline $\begin{array}{l}\text { AMICUS Girls' } \\
\text { Restorative Program } \\
\text { (RADIUS) }\end{array}$ & $\begin{array}{ll} & \begin{array}{l}\text { Mayores índic } \\
\text { optimismo, m }\end{array} \\
\text { Es un programa de Justicia restaurativa que } \\
\text { proporciona a las chicas éxito, en la transición } \\
\text { completa a la comunidad. } & \begin{array}{l}\text { mayores índice } \\
\text { los demás, me } \\
\text { familiares, pers } \\
\text { relaciones entre } \\
\text { disminución } \\
\text { destructivos. }\end{array}\end{array}$ & $\begin{array}{l}\text { es de auto-conciencia, de defensa, } \\
\text { hadurez y motivación, una mejor } \\
\text { de los impactos de sus acciones, } \\
\text { es de compasión y cuidados hacia } \\
\text { ejora de las relaciones personales y } \\
\text { sonal de libertad vigilada y mejores } \\
\text { e los grupos de iguales. Así como la } \\
\text { de comportamientos auto- }\end{array}$ \\
\hline
\end{tabular}


GÉNEROS -Multidisciplinary Journal of Gender Studies, 5(1) 872

\begin{tabular}{|c|c|c|}
\hline Programas & Descripción & Observaciones \\
\hline $\begin{array}{l}\text { Girls and Boys Town SS } \\
\text { Center for Girls. }\end{array}$ & $\begin{array}{l}\text { Es un programa residencial de corto plazo basado } \epsilon \\
\text { el modelo de enseñanza-aprendizaje familiar y } \mathrm{v} \\
\text { tratamiento individualizado. }\end{array}$ & $\begin{array}{l}\text { Se disminuyeron los factores de riesgo desde el } \\
\text { momento en que entró en el programa. Los } \\
\text { mejores predictores de la reincidencia fueron las } \\
\text { evaluaciones realizadas por el personal del } \\
\text { programa y el número de una chica de arrestos } \\
\text { anteriores. }\end{array}$ \\
\hline Girls Circle & $\begin{array}{l}\text { Orientado a mejorar el bienestar físico y } \\
\text { psicológico, conectándolos con otros. }\end{array}$ & $\begin{array}{l}\text { Mejoras significativas en la imagen del cuerpo, la } \\
\text { autoeficacia y el apoyo social percibido, sin } \\
\text { diferencias por raza, edad o ubicación. }\end{array}$ \\
\hline El programa GEMS & $\begin{array}{l}\text { Es un programa de tutoría para niñas involucradas } \\
\text { de } \\
\text { Justicia juvenil. }\end{array}$ & $\begin{array}{l}\text { Aluden cambios deseados en el comportamiento } \\
\text { antisocial y en competencias sociales. }\end{array}$ \\
\hline PACE & $\begin{array}{l}\text { Es un programa de tratamiento individualizado } \\
\text { con visitas al hogar para ellas y sus familias. }\end{array}$ & $\begin{array}{l}\text { Disminución en el consumo de drogas y alcohol } \\
\text { después de salir del programa a largo plazo, sin } \\
\text { embargo no disponía de grupo de control. }\end{array}$ \\
\hline
\end{tabular}

Fuente: Elaboración propia a partir de Kumpfer et al (2008). 


\section{Pozo, Vives \& Orte - Prevención de Drogodependencias}

Los resultados de las evaluaciones de los programas específicos de género se deben en parte a las medidas, métodos, estrategias de investigación, variables y su desarrollo relativamente reciente. Zahn et al (2008) encontraron en su revisión de programas basados en evidencias científicas que realmente existen pocos hallazgos basados en metodologías rigurosas. De los 62 programas que identificaron sólo 18 tenían una evaluación considerada como validada. Tan sólo 9 estaban destinados para las chicas, concluyendo que estas evaluaciones ofrecían evidencias mixta sobre la efectividad de este tipo de programación, pero no mostraban evidencias a largo plazo. Tal y como hemos visto anteriormente, la poca información sobre los enfoques y programas sensibles al género y adecuados para la prevención, es consecuencia o viene motivado por la escasa investigación en factores etiológicos según el género ${ }^{9}$. De hecho, ChesneyLind, Morash, y Stevens (2008) concluyen después de una evaluación de 8 programas específicos para chicas que el conocimiento que se dispone de lo que funciona para las niñas ha tenido pocos avances significativos desde que Lipsey (1992) revisara la literatura disponible y demostrara la escasa evaluación de impacto basada en evidencias científicas. De hecho, las evaluaciones realizadas de los distintos programas preventivos señalan que el 35\% de los programas sólo sirvió a varones (42\% niños atendidos). Sólo el $2 \%$ de los programas sirvió a niñas, (del $6 \%$ destinado a niñas). Una revisión de programas de experiencias exitosas referenciada por la Oficina de Justicia Juvenil y Prevención de la Delincuencia, describen que son 24 los programas que se refieren de forma específica a niños y sólo 2 a niñas (Howell, 2003). De los 62 programas descritos en un estudio efectuado por Bloom et al (2002), el programa más común era un programa de prevención de la delincuencia, seguida por el grupo de tratamiento e institucional. Casi todos fueron mixtos en un (77\%) y sus usuarios/as eran mayormente varones hispanos, caucásicos/as y afroamericanos. De los programas analizados 7 fueron residenciales, mientras que 39 eran no residenciales o programas ambulatorios, 16 eran mixtos (residenciales y no residencial). Además casi la mitad de los programas indicaron que su duración fue variable en el tiempo. La mayoría tenían incorporados evaluaciones psicosociales de sus usuarios/as; aunque pocos tenían servicios de cuidado de niños/as u ofrecían tratamiento residencial para madres y dependientes, pero casi todos aceptaron a mujeres embarazadas, y casi la mitad ofreció clases para padres. 
La mayoría de los programas informaron que proporcionaron asesoramiento y habilidades de formación, así como otros servicios generales, pero menos de dos tercios de los programas declararon ofrecer tratamiento específico para el abuso de sustancias en las jóvenes. La mayoría de los programas informaron que proporcionaban atención individualizada, familiar y con grupos de iguales, y más de tres cuartas partes de los programas ofrecían asesoramiento grupal atendiendo al género. Dos tercios de los programas tenían apoyo educativo o tutorial basado en el desarrollo de habilidades sociales o comunicativas.

\section{Apuntes y recomendaciones finales}

A pesar de este reciente interés por incorporar a las chicas en la investigación y la evaluación de programas preventivos, inclusivos y sensibles al género, muy poco se sabe acerca de las intervenciones específicas en el trabajo con las chicas. La literatura revela que son escasos los que han desarrollado programas con enfoque de género y menos aún los programas preventivos que han publicado sus resultados teniendo en cuenta el género. Existen por lo tanto pocos programas para las niñas y poca literatura sobre la eficacia de los diversos enfoques (Greene, Peters, y asociados, 1998). Además, son muy reducidas las evaluaciones de los programas que se centren exclusivamente en las jóvenes. La literatura y la investigación han concentrado sus esfuerzos principalmente en los varones jóvenes, y poco se sabe acerca de la aplicabilidad de estas intervenciones para ellas. Muchas de las evaluaciones de los programas publicados describen la proporción de niñas incluidas en su muestra pero no realizan un análisis de las diferencias y las similitudes en sus resultados basados en el género. Para ello es imprescindible conocer en primer lugar, que los caminos hacia el consumo de sustancias son diferentes en niñas/os y jóvenes, y cómo las niñas se enfrentan a situaciones vitales diferenciales. Es urgente investigar y realizar el seguimiento en el consumo de drogas y de los factores de vulnerabilidad entre aquellas jóvenes que puedan correr un riesgo significativamente mayor de desarrollar problemas crónicos con las drogas e incorporar estrategias selectivas de intervención dirigidas a responder las necesidades de los grupos más vulnerables. Según la literatura hemos visto que muchos de los servicios existentes no cumplen con las necesidades 
reales de las potenciales consumidoras o delincuentes juveniles. Los distintos servicios de investigación e intervención primaria secundaria y terciaria y sus distintos/as profesionales no están preparados/as para responder a las necesidades específicas de las niñas o jóvenes que participan o están en riesgo de convertirse o involucrarse tanto en el sistema de justicia juvenil como en el consumo de tóxicos (Rosenbaum, 2002). Existen numerosas limitaciones para el tratamiento, siendo el más importante el de la necesidad de financiación, seguido por la falta de formación ${ }^{10}$ e información específica. Las mujeres jóvenes y niñas requieren de personal y de formación especializada, en términos de relación y habilidades de comunicación, diferencias de género en el inicio, abuso y consumo continuado de sustancias tóxicas, abusos sexuales y polivictimización itinerarios delincuenciales, educativos, etapas de desarrollo y procesos vitales de la adolescencia femenina, programas disponibles, referentes de buenas prácticas, ubicaciones adecuadas y limitaciones. Requiere personal especializado formado ${ }^{9}$ en el abuso de sustancias, especialmente en términos de relación y de comunicación a lo largo de las etapas de desarrollo de la adolescencia femenina, y de los distintos programas basados en evidencias científicas disponibles y apropiados además de considerar sus respectivas limitaciones. La American Bar Association así como la National Bar Association (2001) entre otros añadieron la necesidad de desarrollar programas y servicios para atender a sus necesidades particularidades y específicas para mujeres jóvenes. Estos programas deben de ser respetuosos/as y valorar la perspectiva y la experiencia o desarrollo femenino apostando por lo cognitivo-conductual. Además de potenciar la autonomía para alcanzar su máximo potencial. La programación eficaz orientada a las niñas y las mujeres debe ser moldeada y adaptada a sus situaciones y problemas reales. Sin embargo, Bloom et al (2002), señalaron que la dirección específica sobre cómo lograr estos objetivos no es fácilmente evidente a partir de la literatura existente. Los escasos programas eficaces para las niñas y las mujeres deben ser adaptados a su situación problemática y su realidad contextual. Es necesario el desarrollo de una aproximación teórica al tratamiento que sea sensible al género y aborde las realidades de la vida de las niñas. Un enfoque integral debe establecer vínculos y referencias a los programas comunitarios. Estos programas no deben de ser muy ambiciosos y sus servicios deben de contemplar la edad y 
reconocer las diferentes necesidades dentro de las etapas de desarrollo de la niñez, la adolescencia, y la adultez temprana. Las expertas recomiendan más investigaciones y programas teniendo en cuenta el género y la cultura, y que respondan a sus realidades vitales, su identidad étnica y de género, y al desarrollo evolutivo de las mujeres jóvenes o niñas (Chesney-Lind \& Freitas 1999). Y todo ello debe de ser considerado y desarrollado. Por lo tanto la atención debe centrarse en la edad o proceso madurativo del individuo, sus necesidades y fortalezas. Algunas de las propuestas en prevención primaria como secundaria son; La normalización social del alcohol y drogas del colectivo femenino, hace que se requiera de una mayor atención tanto a la publicidad dirigida a estas, y los patrones de consumo de alcohol en niños y niñas/jóvenes. Para ello es necesario revisar los anuncios de tabaco y alcohol que se dirigen a los/las jóvenes a través de las preocupaciones acerca de la apariencia y el deseo de popularidad y éxito. Es por ello que debería de haber un replanteamiento sobre cómo podría trabajarse estas cuestiones en las campañas y los mensajes publicitarios considerando el género. En segundo lugar se debería de reconsiderar ¿Cómo se podría llegar a las niñas y mujeres jóvenes en diferentes niveles de desarrollo, o de riesgo entre otros?. Las tendencias descritas en el inicio de consumo de sustancias especialmente en las jóvenes indican que la transición de educación secundaria a bachillerato es una etapa fundamental para el desarrollo de los programas de prevención en el consumo. En cualquier caso las estrategias para la intervención generales con niñas o mujeres jóvenes deben de considerar las características personales (Sharpe \& Gelsthorpe, 2009), actitudes y experiencias de la infancia incluyendo los traumas, el grupo de iguales y la escuela; influencias de la familia, la cultura y la comunidad, y las influencias sociales, como la publicidad en los medios de comunicación. Por otra parte las conexiones positivas con adultos y grupo de iguales, parejas sanas y personas de referencia o apoyo son los factores de protección para niñas y mujeres jóvenes. Anteriormente hemos visto las interrelaciones entre salud mental, consumo de sustancias y experiencias de violencia y trauma están estrechamente relacionados con las niñas y las mujeres jóvenes. Es preciso indagar más en cómo fortalecer ambientes seguros y positivos en el hogar, la escuela, entre los compañeros/as y la comunidad. Según autores/as como Hubbard (2008), identificaron de la literatura distintos elementos que podrían ser utilizados para desarrollar programas efectivos 
para las niñas y jóvenes; como la evaluación procesual por objetivos, la retroalimentación, la medición regular del cambio, el riesgo/necesidades y la reincidencia. Son urgentes, entre los/as que intervienen y los/as que investigan o teorizan, y entre los distintos países, el intercambio continuado de experiencias exitosas o de buenas prácticas en intervenciones efectivas. Es necesario incorporar la evaluación de los resultados al diseño del proyecto. Así como las estrategias selectivas de prevención del consumo de drogas. Se hace imprescindible establecer una relación de ayudacolaboración desde una perspectiva de género cognitivo-conductual y promover pautas de vida saludables. Las terapias y tratamientos de modificación de conducta deben de incluir competencias para la formación, la defensa propia, la recaída, la asertividad, mejora de la autoestima, estrategias de prevención, empoderamiento y actividades físicas. Además, de la resiliencia personal, crecimiento o desarrollo positivo, la construcción de relaciones interpersonales positivas, arte-terapia, actividades recreativas, terapéuticas (individuales, familiares, la comunidad, el modelado, refuerzo positivo y los procesos o dinámicas grupales. Resulta necesario el seguir reconociendo las diferencias de género y desarrollar programas de mantenimiento o de continuidad; considerando sus necesidades específicas y de desarrollo evolutivo, factores de riesgo y resiliencia, sensible a las diferencias culturales y con evaluaciones periódicas, personal cualificado y especializado y además debe proporcionar recursos, cuidados y apoyos posteriores en la comunidad. Es preciso prestar especial atención a la cultura organizacional de gestión de recursos humanos y a la comunicación interinstitucional basado fundamentalmente en técnicas de resolución de conflictos, procedimientos estructurados de aprendizaje permanente $\mathrm{y}$ específica en habilidades sociales y terapéuticas además del uso efectivo de la autoridad y lo motivacional. Por último apuntar que se necesitan sin duda intervenciones o estrategias a distinto nivel: informativas, educativas, de protección y de control alternativas, reducción de daños en diversos ámbitos tales como el escolar, familiar, comunitario, etc ${ }^{12}$. Por lo que los diferentes autores/as citados recomiendan realizar más estudios basados en evidencias empíricas y efectividad considerando el discurso y las voces especialmente de las jóvenes. Se necesita mucha más información con respecto al proceso y desarrollo evolutivo y de desarrollo de las jóvenes y sus factores de riesgo/protección (Schinke, Fang \& Cole, 2008), así como a la eficacia y 
eficiencia de los programas de intervención y prevención para que el sistema de protección, Justicia juvenil, servicios sociales, responsables políticos y los distintos profesionales de intervención e investigación puedan ofrecer servicios apropiados sensibles al género y a la cultura. Sin duda todas las instituciones y organismos tanto de investigación como de intervención (primaria, secundaria o terciaria) se enfrentan a un gran desafío con el número creciente de mujeres jóvenes consumidoras que aparecen en el sistema de justicia juvenil y el adulto.

\section{Notas}

${ }^{1}$ Baskin y Sommers (1993) apoyaron la idea de cómo las chicas han sido "largamente excluidas de la delincuencia", además de seguir considerando los viejos roles de la mujer criminal contemporánea, donde el crimen estaba muy masculinizado (como por ejemplo los delitos violentos) o feminizado (la (s) fuga (s) y prostitución).

${ }^{2}$ Para más información puede consultarse "Girls, delinquency and juvenile justice" de Chesney-Lind y Shelden (2004).

${ }^{3}$ Sobre estas cuestiones ya incidiremos más adelante.

${ }^{4}$ Reconocer los diferentes perfiles ayudaría a identificar subgrupos de mujeres para quienes los programas particulares son eficaces (Zahn y otros 2009).

${ }^{5}$ Es preciso señalar que algunos de los autores/as se encuentran a caballo entre estos posicionamientos.

${ }^{6}$ Así mismo destacar que estos programas de prevención del tabaquismo están destinadas a la población general y que las chicas con problemas de consumo importantes e inmersas en procesos delincuenciales comparten "otros problemas" que los de la población de mujeres jóvenes y chicas en la comunidad. Por lo tanto el abordaje preventivo de drogodependencias debe de ser holístico y contemplar una serie de elementos significativos para este colectivo particular de jóvenes.

${ }^{5}$ Es preciso señalar que muchas de las chicas implicadas en contactos reiterados con el sistema de justicia juvenil tienen familias muy disruptivas (según los estudios que han comparado chicas y chicos, las familias de ellas son más problemáticas o no tienen). Por lo que esta aproximación tendría sus limitaciones.

${ }^{8}$ Debido a que la depresión temprana en la adolescencia se vuelve más frecuente entre las niñas que entre los muchachos, y esta discrepancia es aún más pronunciada en la adolescencia.

${ }^{9}$ Los existentes han tomado fundamentalmente como referencia a jóvenes que han cometido infracciones, faltas o delitos.

${ }^{10}$ Es muy escasa la investigación sobre las actitudes y experiencias de profesionales que trabajan con las chicas. Entre la investigación que existe, se observa que muchas personas que trabajan en el sistema de justicia juvenil sostienen que las niñas son más difíciles de trabajar con respecto a los varones (Belknap, Holsinger, \& Dunn, 1997).

${ }^{11} \mathrm{La}$ investigación también indica que los profesionales tienen una falta de experiencia y de conocimientos sobre el abuso sexual pese a que muchas chicas delincuentes experimentar este 
tipo de abuso. El trabajo con las mujeres requiere una formación especializada Bloom, Owen, Piper Deschenes y Rosenbaum (2002).

12 Todos ellos deben de garantizar el acceso a los servicios específicos de violencia contra las mujeres, y a los de salud sexual y reproductiva).

\section{Referencias}

Adelberg \& Currie (1987). Too few to count: Canadian women in conflict with the law. En A. Morris, with the assistance of L. Gelsthorpe (December 1980) Women and crime papers presents to the Crowood Round table conference. Cropwood conference series, 13. University of Cambridge Institute of Criminology.

AMERICAN BAR ASSOCIATION \& NATIONAL BAR ASSOCIATION. (2001). "Justice by Gender: The Lack of Appropriate Prevention, Diversion and Treatment Alternatives for Girls in the Justice System". Washington, D.C.: American Bar Association and the National Bar Association.

Baskin, D. \& Sommers, I. B, (1993). "Females' Initiation into Violent Street Crime.' Justice Quarterly, 10, 559-581. doi: 10.1080/07418829300092021

Belknap, J. \& Holsinger, K. (1998). An overview of delinquent girls: How theory and practice failed and the need for innovative changes. En R.T. Zaplin (Ed.), Female offenders: Critical perspectives and effective interventions. Gaithersburg, MD: Aspen Publishers.

Belknap, J. (2001). The invisible woman: Gender, crime, and justice, Second Edition. Belmont, CA: Wadsworth.

Blake, S. M., Amaro, H., Schwartz, P. M. \& Flinchbaugh, L. J. (2001). A review of substance abuse prevention interventions for young adolescent girls. Journal of Early Adolescence, 21(3), 294-324. doi: 10.1177/0272431601021003003

Bloom, B.E., Owen B., Piper Deschenes, E. \& Rosenbaum, J. (2002) Improving Juvenile Justice for Females: A Statewide Assessment in California. Crime \& Delinquency, 48(4), 526-552. doi: $10.1177 / 001112802237128$

Brown, L.M. (2003). Girlfighting: Betrayal and rejection among girls. New York: New York University Press. 
Burt, M.R; Glynn, T.J. \& Sowder, D.J. (1979). Psychosocial Characteristics of Drug Abusing Women. NIDA. Research Monograph. DHEW Publication No. ADM 80-197. Rockville, Maryland : U.S.GPO.

Cameron, H. \& Telfer, J. (2004). Cognitive-behavioural group work: Its application to specific offender groups. Howard Journal of Criminal Justice, 43, 47-64. doi: 10.1111/j.1468-2311.2004.00310.x

Cullen, F.T. \& Gendreau, P. (2000). Assessing correctional rehabilitation: Policy, practice, and prospects. En J. Horney (Ed.) Policies, processes, and decisions of the criminal justice system; criminal justice (Vol. 3, pp.109-175). Washington, DC: National Institute of Justice/ NCJRS.

Chesney-Lind, M. \& Shelden, R.G. (1997). Girls, delinquency, and the juvenile justice system ( $2^{\text {nd }}$ ed.), Belmont, CA:Wadsworth Publishing Company Community Research Associates. Juvenile Female Offenders: A Status of the States Report. Washington, DC: U.S. Department of Justice, Office of Justice Programs, Office of Juvenile Justice and Delinquency Prevention.

Chesney-Lind, M. \& Freitas, K. (1999). "Working with Girls: Exploring Practitioner Issues, Experiences and Feelings". A report of the Hawaii Girls Project, 4. Manoa, HI: The Center for Youth Research, Social Science Research Institute, University of Hawaii at Manoa.

Chesney-Lind, M. (1999). Challenging girls' invisibility in the juvenile court. The Annals of the American Academy of Political and Social Science, 564,185-202.

Chesney-Lind, M. (2000). What to do about Girls. En M. McMahon (Ed). Assessment to Assistance: Programs for Women in Community Corrections. (pp. 139-170) Lanham, MD: American Correctional Association.

Chesney-Lind, M. \& Shelden, R.G. (2004). Girls, delinquency and juvenile justice ( $3^{\mathrm{a}}$ Ed.) Belmont:Wadsworth

Chesney-Lind, M., Morash M. \& Stevens T. (2008). Girls' Troubles, Girls' Delinquency, and Gender Responsive Programming: A Review. The Australian and New Zealand Journal of Criminology 41 (1),162-89. doi: 10.1375/acri.41.1.162

Dembo, R., Williams, L., Schmeidler, J. \& Christensen, C. (1993). Recidivism in a cohort of juvenile detainees: A3 1/2 year follow-up. International Journal of the Addictions, 28, 631-658. 
Gottfredson, D. C. \& Kruttschnitt, C. (2008). Violence by Teenage Girls: Trends and Context: Office of Justice Programs, Office of Juvenile Justice and Delinquency Prevention, Girls Study Group.

Gaarder, E., Rodríguez, N. \& Zatz, M.S. (2004). Criers, Liars, and Manipulators: Probation Officers' Views of Girls. Justice Quarterly, 21 (3), 547-578. doi: 10.1080/07418820400095901

Gelsthorpe, L. (1989) Sexism and the Female Offender. Cambridge Studies in Criminology. Aldershot: Gower.

Gendreau, P. (1996). The principles of effective intervention with offenders.

En A. Harland (Ed.) Choosing correctional options that work: Defining the demand and evaluating the supply (pp. 117-130). Thousand Oaks, CA: Sage.

Greene, P. \& Associates (1998). Guiding principles for promising female programming: An inventory of best practices. Washington, DC: U.S. Department of Justice, Office of Justice Programs, Office of Juvenile Justice and Delinquency Prevention.

Glynn, T.J., Leukefeld, C.G. \& Ludford, J.P., eds. (1983) Preventing Adolescent Drug Abuse: Intervention Strategies. National Institute on Drug Abuse Research Monograph 47. DHHS Pub. No. (ADM)83-1282. Washington, DC: Supt. of Docs., U.S. Govt. Print. Off

Glynn, T.H., Wallenstein Pearson, J.H \& Sayers, M. (1983). (Eds.) Women and Drugs, NIDA Research Issues 31. Rockville, Md: National Institute on Drug Abuse, U.S. Department of Health and Human Services. Pearson $\&$ M. Sayers (Editors).

Howell, J. (2003). Preventing and Reducing Juvenile Delinquency: A Comprehensive Framework. Thousand Oaks, Calif.: SAGE.

Hubbard, D.J. \& Matthews, B. (2008). Reconciling the differences between the -Gender- Responsivell and the - What Worksll literatures to improve services for girls. Crime and Delinquency, 54, 225-258. doi: $10.1177 / 0011128706296733$

Huizinga, D. (1997). Over-Time Changes in Delinquency and Drug Use: The 1970's to the 1990's. Unpublished report. Washington, DC: Office of Juvenile Justice and Delinquency Prevention.

Kumpfer, K. L., Alvarado, R. et al (2003). Family interventions for the prevention of drug abuse. American Psychologist 58(6-7), 425-432. 
Kumpfer K L., Smith P. \& Summerhays J.F., (2008) AWakeup Call to the Prevention Field: Are Prevention Programs for Substance Us Effective for Girls? Substance Use \& Misuse. Utah, USA.

Lipsey, M.W. (1992). Juvenile delinquency treatment: A meta-analytic inquiry into the variability of effects. En T.A. Cook, H. Cooper, D.S. Cordray, H. Hartmann, L.V. Hedges, R.J. Light, T.A. (Eds.) MetaAnalysis for explanation: a Casebook. (pp 83-127). New York, NY: Russell Sage Foundation.

Louis T.A. \& Mosteller, F. (Eds.) (1992). Meta-analysis for explanation: A casebook (pp. 83-126). New York: Russell Sage.

National institute on drug abuse. (1979). Problems of Drug Dependence: Proceedings of the 41st Annual Scientific Meeting, the Committee on Problems of Drug Dependence, Inc.

National institute on drug abuse. (2002). Study quantifies cost-benefit of family interventions designed to prevent adolescent alcohol use. NIDA News Release. Available at www.drugabuse.gov.

Orte, C. \& GIFES. (2005). Los programas de prevención de drogas centrados en la familia: una visión desde la investigación y la práctica. Revista Proyecto (53),14-17.

Orte, C. \& GIFES. (2005). Una investigació educativa sobre un programa de competència familiar. En M. March (Dir.) Anuari de l'Educació de les Illes Balears (pp 284-295). Palma: Fundació Guillem Cifre de Colonya.

Rosenbaum, J. (2002). Improving Juvenile Justice for Females: A Statewide Assessment in California. Crime \& Delinquency, 48(4), 526.

Rumgay, J. (1998). Crime, Punishment and the Drinking Offender. Basingstoke: Macmillan.

Schinke, S.P., Fang, L. \&. Cole, K.C.A (2008). Substance use among early adolescent girls: Risk and protective factors. Journal of Adolescent Health, 43(2) 191-194.

Sharpe G. \& Gelsthorpe L. (2009). Engendering the Agenda: Girls, Young Women and Youth Justice. Youth Justice, 9(3), 195-208. doi: 10.1177/1473225409345098

Worrall, A. (2001). Girls at Risk? Reflections on Changing Attitudes to Young Women's Offending, Probation Journal, 48(2), 86-92. doi: $10.1177 / 026455050104800203$ 
883 Pozo, Vives \& Orte - Prevención de Drogodependencias

Yin, R. K. \& Ware, A. J. (2000). Using outcome data to evaluate community drug prevention initiatives: Pushing the state-of-the-art. Journal of Community Psychology, 28, 323-338. doi: 10.1002/(SICI)15206629(200005)28:3<323::AID-JCOP7>3.0.CO;2-L

Zahn, M.A., Hawkins, S.R., Chiancone, J. \& Whitworth A. (2008). The Girls Study Group-Charting the Way to Delinquency Prevention for Girls. U.S. Department of Justice, Office of Juvenile Justice and Delinquency Prevention.

Zahn, M.A., Day, J.C., Mihalic, S.F. \& Tichavsky, L. (2009). Determining What Works for Girls in the Juvenile Justice System. Crime \& Delinquency, 55(2), 266-93. doi: 10.1177/0011128708330649 
Anexo 1:

Factores de riesgo y de protección relevantes en niñas y chicas jóvenes

\section{Factores de riesgo}

- Estresores graves (ej pobreza, muerte, violencia, y un patrón multigeneracional de encarcelamiento).

- Familias monoparentales (habitualmente una figura femenina asume la responsabilidad de los cuidados y crianzas),

- Antecedentes familiares (drogodependencias y contacto con el sistema de justicia).

- (Poli)Victimización: -Fracaso escolar generalizado -Depresión y la ansiedad (desarrollo de trastornos derivados).

- El barrio(s) (con altos niveles de pobreza, criminalidad, desempleo, violencia, drogodependencia, institucionalización de sus miembros entre otros).

- Nivel de acceso inferior (de las chicas) a los programas comunitarios.

- Pubertad temprana

- Itinerario(s) de vulnerabilidad a edades más tempranas con fracasos escolares embarazos, entre las edades de 12 y 15 años.

- Embarazo o la maternidad adolescente. Actividad sexual temprana.

- Actividad de consumo temprana.

\section{Factores de protección}

- Controles escolares o la familiares.

- Control o supervisión de los horarios de llegadas y de salidas (sobre todo después de la escuela).

- Apoyo, estímulos o influencia "positiva" (para abstenerse de consumir).

- Comunicación fluida y en términos de cuidados de un adulto.

- Confianza.

- Función y actitud protectora.

- Temor a las consecuencias de un posible consumo.

- "Madre" informada del paradero de su hija(s)(así como la de sus compañeros/as).

- La posibilidad de que sus hijas se puedan poner en contacto con su madre.

- Estímulos familiares positivos para abstenerse de consumir. 


\section{Factores de riesgo}

- La asociación con muchachos "desviados".

- Trastornos alimentarios depresión en las jóvenes baja autoestima acompañado de un aumento en la depresión e intentos de suicidio para las adolescentes

- Estrechas relaciones entre el consumo de alcohol de mujeres jóvenes y el desarrollo de depresión, la obesidad, y trastornos (salud mental).

- Distorsión en su autopercepción y quieren ser aceptadas, respetadas y sentirse atractivas.

- Válvula de escape eficaz al dolor de las relaciones familiares disfuncionales, maltrato familiar, el aislamiento, enajenaciones, baja autoestima, y la falta de apoyo social.

- Trastorno de estrés postraumático y depresivo.

- Hechos frustrantes de su vida, la victimización temprana, la pérdida de sus hijos, para escapar del dolor y la propia realidad, comienzan a consumir.

- Depresión y ansiedad, bajo nivel de autoestima, dificultades de comportamiento emocional, tendencias suicidas, trastornos alimenticios, dieta entre otros.

\section{Factores de protección}

- Disponer de reglas familiares claras y concisas sobre el uso nocivo de sustancias tóxicas.

- Actitud protectora (y el miedo o temor a iniciar un posible consumo).

- Fortalecer el vínculo de las familias con las escuelas para promocionar el éxito (escolar).

- Percepción positiva del entorno escolar. 
GÉNEROS-Multidisciplinary Journal of Gender Studies, 5(1) 886

Rosario Pozo Gordaliza Universitat de les Illes Balears

Carmen Orte Universitat de les Illes Balears

Marga Vives Universitat de les Illes Balears

Contact address: Department of Education, Universitat de les Illes Balears, Edifici Guillem Cifre de Colonya. Cra, de Valldemossa Km. 7'5. Palma (Illes Balears. Spain)

E-mail address: rosariopozogordaliza@gmail.com 